PHYSICAL REVIEW D 96, 109901(E) (2017)

\title{
Publisher's Note: Constraints on Kähler moduli inflation from reheating [Phys. Rev. D 96, 083522 (2017)]
}

\author{
Sukannya Bhattacharya, Koushik Dutta, and Anshuman Maharana
}

(Received 7 November 2017; published 13 November 2017)

DOI: 10.1103/PhysRevD.96.109901

This paper was published online on 23 October 2017 with an error in the title. The title should read "Constraints on Kähler moduli inflation from reheating." The title has been corrected as of 8 November 2017. The title is incorrect in the printed version of the journal. 